\title{
An Autonomous Navigation Methodology for a Pioneer 3DX Robot
}

Salvador Ibarra Martínez, José Antonio Castán Rocha ${ }^{1}$, Julio Laria Menchaca ${ }^{1}$, Mayra Guadalupe Treviño Berrones ${ }^{1}$, Javier Guzmán Obando ${ }^{1}$, Julissa Pérez Cobos ${ }^{1}$ and Emilio Castán Rocha ${ }^{2}$

1. Computer Science Department, Engineering School, Autonomous University of Tamaulipas, Victoria, Tamaulipas, 87000, Mexico.

2. Electrical and Electronic Department, Technological Institute of Madero City Tampico, Tamaulipas, 89440, Mexico.

\begin{abstract}
Autonomous navigation is a complex challenge that involves the interpretation and analysis of information about the scenario to facilitate the cognitive processes of a robot to perform free trajectories in dynamic environments. To solve this, the paper introduces a Case-Based Reasoning methodology to endow robots with an efficient decision structure aiming of selecting the best maneuver to avoid collisions. In particular, Manhattan Distance was implemented to perform the retrieval process in CBR method. Four scenarios were depicted to run a set of experiments in order to validate the functionality of the implemented work. Finally, conclusions emphasize the advantages of CBR methodology to perform autonomous navigation in unknown and uncertain environments.
\end{abstract}

Key words: Autonomous navigation, pioneer 3DX robot, CBR methodology.

\section{Introduction}

Mobile robot is an integrated system which consisted of environmental perception, dynamic decision and planning. A robot does not possess natural senses like human beings have. Indeed, human beings get information about their surrounding through vision and other natural sensing power. For thus, a mobile robot needs reliable information of the environment before to decide which movement it must do. In this sense, a robot cannot explore an unknown environment unless it is provided with some sensing sources to get information about the environment.

Different kinds of sensors such a sonar, odometers, laser range finders, IMU (inertial measurement units), GPS (global positioning system) and cameras are commonly used to make a robot capable of sensing a wide range of scenarios. To execute a free navigation in an indoor environment, a robot should perform

\footnotetext{
Corresponding author:Salvador Ibarra Martínez, doctor, research fields: intelligent systems, autonomous mobile robots and automatic learning techniques. E-mail: sibarram@uat.edu.mx.
}

some maneuvers to avoid crash with objects and walls. To perform such maneuvers, the robot must be capable to handle data about the distance between him and the surrounding obstacles. Traditional global navigation mode is difficult to apply to this case, which consists of a perception of the environment. In reactive navigation mode, the adaptation of local path planning based on sonar data will realize the navigation task in unknown and complex scenario [1-2]. However, it is easy to fall into local traps due to the lack of global planning, causing the repeated paths and the failed navigation. Some recent works in the literature are devoted to study and solve the indoor navigation problem from different points of view.

In Ref. [3], an obstacle avoidance behavior based fuzzy logic control and follow walls to realize the navigation in an unknown and complex environment is presented. Using FSM (finite state machine), the navigation status of mobile robot transfer when the information of environment changes, and a corresponding strategy is chosen to realize the navigation task. This algorithm can effectively solve 
the local trap problems in traditional mobile robot navigation strategy. Some experiments are presented on the Pioneer 3DX mobile robot and good results are obtained.

Reference [4] presents an approach for robot exploration in large-scale unknown environment by concurrent and incremental construction of a hybrid environment model, which is built on top of a RBPF-SLAM (rao blackwellized particle filter-simultaneous localization and mapping) RBPF-SLAM system. In this work, SLAM technique for robot exploration is based on laser scan-matching and RBPF. The model of the unknown environment is structured as a hybrid representation, both topological and grid-based, and it is incrementally built during the exploration process.

For instance, the author of reference [5] proposes a spiking-neural-network-based robot controller inspired by the control structures of biological systems. Information is routed through the network using facilitating dynamic synapses with short-term plasticity. The network self-organizes to provide memories of environments that the robot encounters.

A Pioneer robot simulator with laser and sonar proximity sensors is used to verify the performance of the network with a wall-following task, and the results are presented.

The work described in Ref. [6] shows how a ros-based control system is used with a Pioneer 3DX robot for indoor mapping, localization, and autonomous navigation. Mapping of different challenging environments is presented in this work. Moreover, some factors associated with indoor environments that can affect mapping, localization, and automatic navigation are also presented.

When dealing with dynamic changing environments, behaviour-based systems need to adapt. However, changes are difficult to model and predict. The main drawback of modeling is the use of parameters to characterize kinematics and dynamics [7]. These parameters need to be optimized for each specific problem, especially if different robots are used. Furthermore, if the robot is affected by physical problems, the same parameter optimization has to be used. Hence, it would be desirable to achieve a behaviour-based scheme able to adapt to changing circumstances without human supervision, allowing the system to work in a different robot after minor changes. In this context, CBR (case-based reasoning) emerges as an alternative for adapting to environment changes. CBR is a learning and adaptation technique to solve current problems by retrieving and adapting past experiences [8]. As demonstrated, when using CBR, there is no need to study the robot kinematics nor the environment [9].

The remainder of this paper is organized as follows. Section 2 introduces the main aspects of the hardware robot and the software used in the experiments. In Section 3, the formalization of the CBR methodology followed for the experimental results given in Section 4. Conclusions are made at last in Section 5.

\section{Robot Platform}

A mobile robot Pioneer 3DX which is a two-wheel differential drive robot is used as experiment platform (Fig. 1). The Pioneer robots are one of the most popular research robot test beds. Because of its models and balanced size combined with reasonable hardware, it is most suitable for in-door navigation.

Multiple sensors are used to overcome the illusion of interference provided by the ultrasonic sensors due to blind spots existing in ultrasonic detection, especially

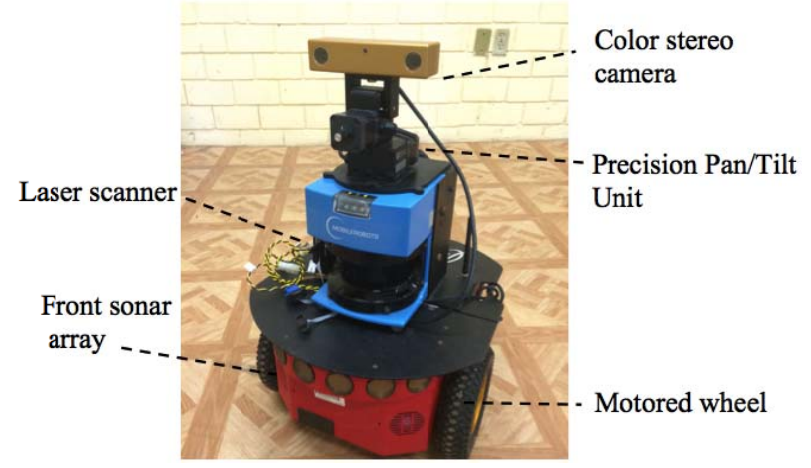

Fig. 1 The Pioneer 3DX robot. 
when theultrasonic sensors and obstacles form incorrect data for the robot. To make the robot fully capable of identification of the objects, two sonar rings with a set of 16 sensors are used. Linux (Debian) on-board computer system is used to implement the proposals of the work. (Fig. 2)

\section{Methodology}

In this paper, cases-based reasoning methodology is used for the robot's navigation task. CBR reuses the knowledge achieved by solving the same problem previously to reason the new one, and then makes adaptation based on the differences to give the solution. Furthermore, the intelligent character is helpful for improving the response ability and making decision more scientific. CBR stores any possibly interesting situation in a case-base in the form of cases. A CBR case is a $N$-dimensional input vector to characterize a given situation and the solution to that situation. The advantage of CBR compared to another techniques, such as neural networks, is that cases in the case-base are explicitly stored. Thus, cases can be easily analyzed to have a clear idea of what the robot has learnt and why it performs a given action. Furthermore, learning through CBR is preferable than neural networks since it is possible to seed the case-base with a-priori knowledge.

\subsection{Case Representation}

Source case is stored in case database and may be reused to settle the target problem. The navigation source case is constructed by inputs obtained by the sensors from the environment and the current navigation strategy, such that:

$$
\text { source_case }_{\mathrm{n}}=\left\{\mathrm{NAV}, \mathrm{d} \_ \text {sensor } 0, \mathrm{~d} \_ \text {sensor }{ }_{1} \ldots, \mathrm{d} \_\right. \text {sens }
$$

$\left.\mathrm{or}_{15}\right\}$

The output of the case consists of the selection of a navigation strategy to the robot (Section 4) leading by the retrieved cases. (Fig. 3)

\subsection{Retrieval Process}

Minor difference among sensor reading may lead to different case. However, these differences usually correspond to same situations. Since it has been proven that it is better to combine discrete and continuous data in CBR systems [10], the problem instance can be improved by discretizing the sensor readings by the direction of the robot's maneuver. Thus, the retrieval process consists of matching all cases $\overrightarrow{\mathrm{R}}$ in the case-base against the current problem $\overrightarrow{\mathrm{C}}$. Obviously, the most similar is selected by evaluating the similarity between cases through an adaptation of the Manhattan distance proposed in Ref. [7] such as follow:

where $W=[\mathbf{w 0}, \mathbf{w} 2, \mathbf{w} 3, \ldots, \mathbf{w} 15]$ is the vector of weights for sensors defined by each navigation system.

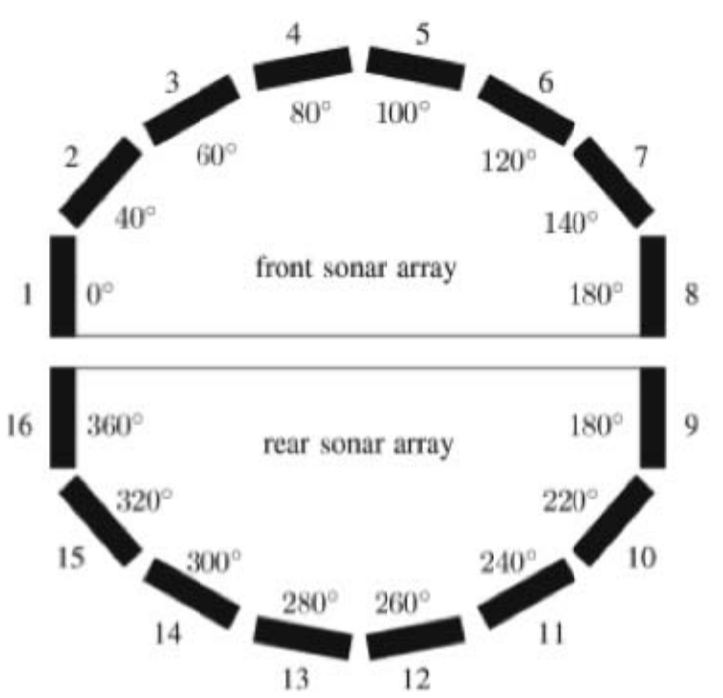

Fig. 2 The Pioneer robot's 16 sonar sensors shown with the angel of each sensor.

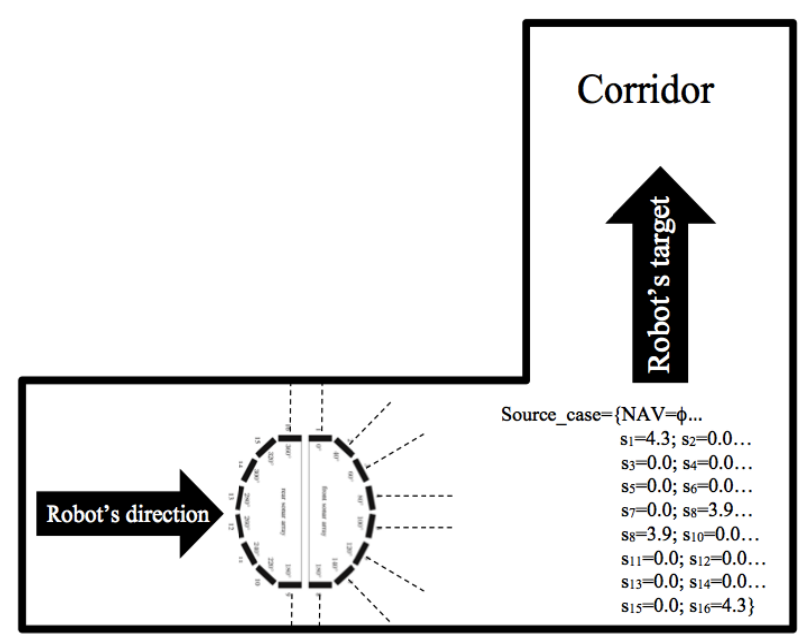

Fig. 3 Scheme of a particular case example. 
For the experimental proposal, the weight plays a relevant role to influence the motion of the robot. Such fact, it is the principal difference with [7].

\subsection{Reuse Process}

In order to use the solution that covers all issues of the local trap problems in an indoor navigation task successfully, the robot adopts the navigation strategy recommended by the retrieval process. Such strategies try to generate a general idea of the environment avoiding that robot constructs a mental state of its position and the configuration of the scenario.

\subsection{Review Process}

For experimental reasons, this phase has not been used in the proposed approach.

\subsection{Retain Process}

According with the basic function of the CBR method, this process is devoted to index new cases to the case-base when the source_case has not an exact match in the case-base.

\section{Navigation Strategies}

A navigation strategy refers to a set of strategies, which allows the mobile robot to obtain the power that must be applied to the encoders of each wheel at any time. The following sections introduce an overview of the four proposed strategies.

(1) $\phi$ Frontal navigation strategy

It is the main strategy. This strategy is predefined as the initial state of the robot when it starts its navigation tasks. The operation of this system is very simple, it applies the same power (pwm $=+80$ ) for both encoders.

(2) $\theta$ Reverse navigation strategy

In some situations, the robot must correct its trajectory due to several aspects (i.e., a corridor, a wall, a loop, etc.). In this sense, one functional strategy is to make a backward movement. This maneuver will allow robot to leave such circumstances to return to the right path. To escape from such situation, the reverse navigation system applies negative values to the encoders of the wheels (i.e., pwm $=-50$ ) for 10 s and then, it calls to the Left_turn navigation or Left_right navigation strategies.

(3) $\varphi$ Left_turn navigation strategy

This complementary but functional strategy is the perfect complement for the navigation system of an autonomous mobile robot. The Left_turn strategy proposes a semi-circular turning towards the left sizes of the robot. To do this, the strategy employs different powers for the robot's encoders (pwmleft_encoder = 20 and pwmright_encoder $=40$ ) for $10 \mathrm{~s}$ and then, it calls to the frontal navigation strategy.

(4) $\omega$ Right_turn navigation strategy

To complete the set of strategies, the right-turn strategy proposes a semi-circular turning towards the right size of the robot. To do this, the strategy employs different powers for the robot's encoders (pwmright_encoder $=20$ and pwmleft_encoder $=40$ ) for $10 \mathrm{~s}$ and then, it calls to the frontal navigation strategy.

\section{Experiments and Results}

\subsection{Experiments Features}

The proposed system has been tested on a Pioneer 3-DX equipped with eight frontal and eight rear sonar sensors in an indoor unknown and changing environment. In order to evaluate the performance of the robots, the test measures the time that a robot can be navigating in the environment without presenting any collision with the objects located in the scenario. Fig. 4 illustrates the four proved scenarios. The dimensions of the room are $7.2 \mathrm{~m} \times 15.8 \mathrm{~m} \times 2.5 \mathrm{~m}$. For experimental reasons, each test is fixed in $5 \mathrm{~min}$ and the number of events in that robot hits against something is controlled in a manual way by the authors. The number of experiments for each scenario is stated in 10 trials. Finally, to evaluate the quantity of cases that were generated during each test and to be able to compare it with other case-base, at the end of each experiment the case-base was restarted. 


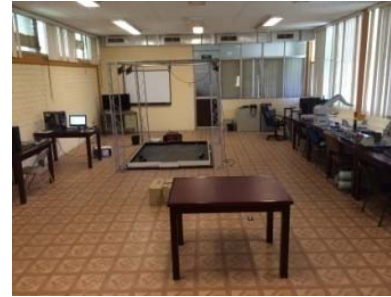

(a)

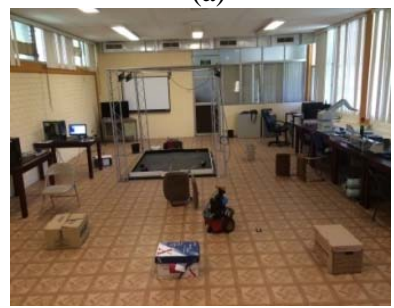

(c)

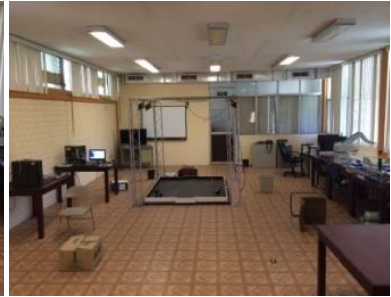

(b)

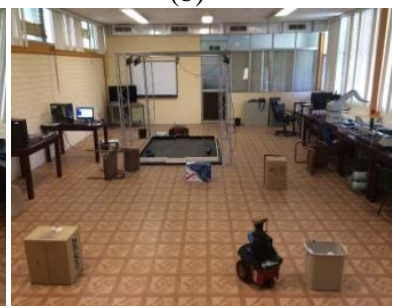

(d)
Fig. 4 Real scenarios used in the experiments:(a) configuration 1; (b) configuration 2; (c) configuration 3 ; (d) configuration 4.

\subsection{Experiment Results}

The experiments depicted in previous sections reach some interesting results related to the capability of a mobile robot to perform autonomous navigation in an indoor unknown environment.

For example, in the scenario 1, the robot reaches at least $76 \%$ of effective decisions from 148,428 decisions. It means that when a robot must implement a particular navigation strategy proposed by the CBR model, such decision was successful for the navigation task. Besides, for this test, the case-base reports an average of 322 cases.

In Table 1 is presented more relevant information of the test is presented. In addition, Fig. 5 shows how the robot's performance increases in almost $37 \%$ through the tests when the case-base has not been restarted.

Otherwise, in the scenario 2, the robot is capable to reach around $78 \%$ of good decisions out $(149,603)$. In this test, the case-base reports an average of 320 cases. The robot's performance in the 10 experiments is compared to emphasize the advantages of CBR model. In Table 2 is presented more relevant information of the testis presented. In short, Fig. 6 shows how the robot's performance increases in almost 38\% through the tests when the case-base has not been restarted. Specifically, the first and the last experiments results were compared.

Meanwhile, the scenario 3 reports that the robot can make efficient decisions in almost $75 \%$ when it must choose a particular movement to avoid a collision. Fig. 7 illustrates the progressive evolution of the robot's decision throughout the experiments. The complete

Table 1 Additional information of scenario1.

\begin{tabular}{lllllll}
\hline Test & \#hits & Cases & $\phi$ & $\theta$ & $\varphi$ & $\omega$ \\
\hline 1 & 21 & 320 & 10,488 & 1,233 & 1,327 & 975 \\
2 & 25 & 317 & 10,445 & 1,257 & 1,419 & 1,128 \\
3 & 18 & 311 & 10,987 & 1,245 & 1,423 & 1,104 \\
4 & 17 & 314 & 11,112 & 1,222 & 1,388 & 1,136 \\
5 & 16 & 328 & 11,293 & 1,203 & 1,232 & 1,042 \\
6 & 18 & 319 & 11,445 & 1,214 & 1,301 & 983 \\
7 & 19 & 323 & 11,821 & 1,033 & 1,287 & 992 \\
8 & 17 & 329 & 11,834 & 1,078 & 1,293 & 971 \\
9 & 19 & 326 & 12,028 & 993 & 1,276 & 982 \\
10 & 16 & 332 & 12,032 & 989 & 1,254 & 963 \\
AVE & 19 & 322 & 113,485 & 11,467 & 13,200 & 10,276 \\
\hline
\end{tabular}

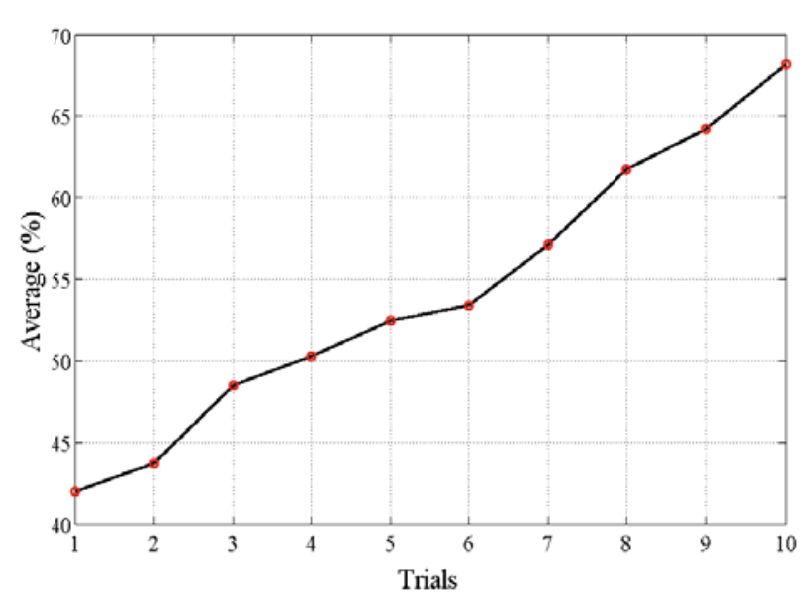

Fig. 5 Progressive evolution of the robot's decisions.

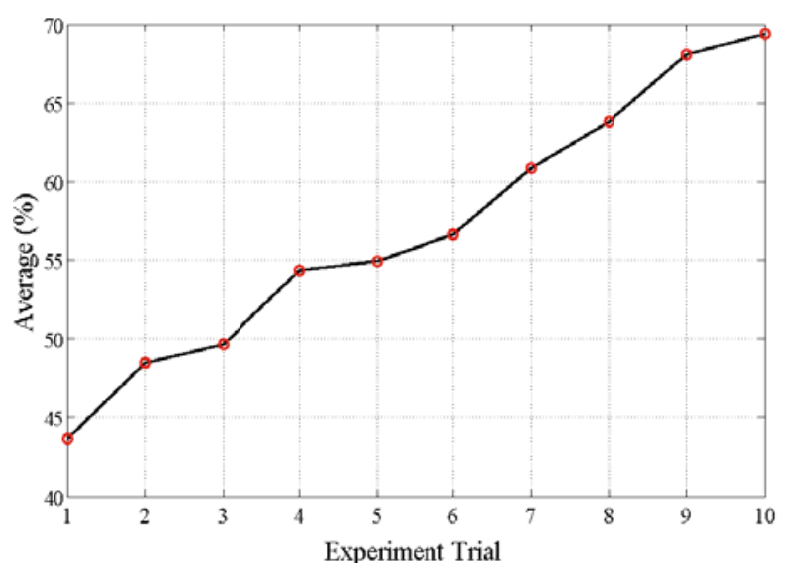

Fig. 6 Accumulated performance of the robot's decisions in 10 episodes. 
Table 2 Additional information of scenario2.

\begin{tabular}{lllllll}
\hline Test & \#hits & Cases & $\phi$ & $\theta$ & $\varphi$ & $\omega$ \\
\hline 1 & 26 & 307 & 10,674 & 1,335 & 964 & 1,092 \\
2 & 20 & 319 & 11,815 & 1,570 & 988 & 1,120 \\
3 & 21 & 319 & 11,784 & 1,354 & 1,043 & 1,114 \\
4 & 23 & 316 & 11,132 & 1,312 & 1,118 & 1,102 \\
5 & 21 & 314 & 11,933 & 1,322 & 1,123 & 1,023 \\
6 & 19 & 331 & 10,874 & 1,309 & 1,208 & 1,003 \\
7 & 19 & 327 & 11,793 & 1,279 & 1,198 & 992 \\
8 & 27 & 311 & 10,455 & 1,257 & 1,234 & 1,114 \\
9 & 19 & 329 & 11,966 & 1,199 & 1,223 & 973 \\
10 & 18 & 332 & 12,003 & 1,201 & 1,231 & 1,173 \\
AVE & 21 & 320 & 114,429 & 13,138 & 11,330 & 10,706 \\
\hline
\end{tabular}

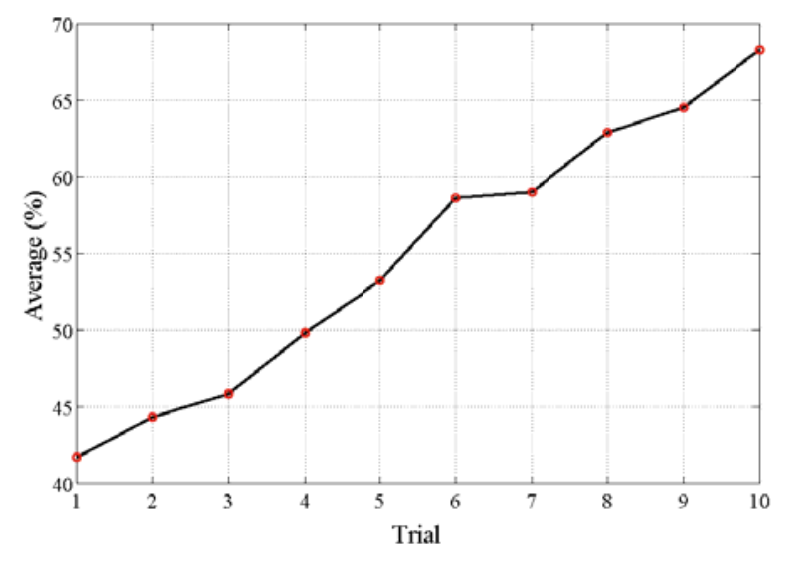

Fig. 7 Progressive evolution of the robot's decisions.

Table 3 Additional information of scenario3.

\begin{tabular}{lllllll}
\hline Test & \#hits & Cases & $\phi$ & $\theta$ & $\varphi$ & $\omega$ \\
\hline 1 & 17 & 326 & 11,764 & 1,086 & 1,143 & 928 \\
2 & 16 & 329 & 11,893 & 989 & 1,117 & 988 \\
3 & 22 & 318 & 10,955 & 976 & 1,123 & 1,103 \\
4 & 19 & 324 & 11,142 & 1,242 & 965 & 1,201 \\
5 & 21 & 319 & 11,557 & 1,213 & 1,132 & 976 \\
6 & 19 & 322 & 11,398 & 1,291 & 1,087 & 1,001 \\
7 & 16 & 332 & 10,332 & 979 & 981 & 999 \\
8 & 17 & 328 & 11,342 & 1,235 & 1,113 & 1,034 \\
9 & 19 & 326 & 11,936 & 1,576 & 1,046 & 1,102 \\
10 & 18 & 324 & 11,984 & 982 & 1,229 & 1,108 \\
AVE & 18 & 324 & 114,303 & 11,569 & 10,936 & 10,440 \\
\hline
\end{tabular}

analyzingresults are summarized in Table 3. Moreover, Fig. 7 shows how the robot's performance increases in almost $40 \%$ through the tests when the casesbase has not been restarted.

Finally, in the scenario 4 , the robot reaches at least $76 \%$ of effective decisions from out $(153,440$ decisions). Fig. 8 illustrates the progressive evolution

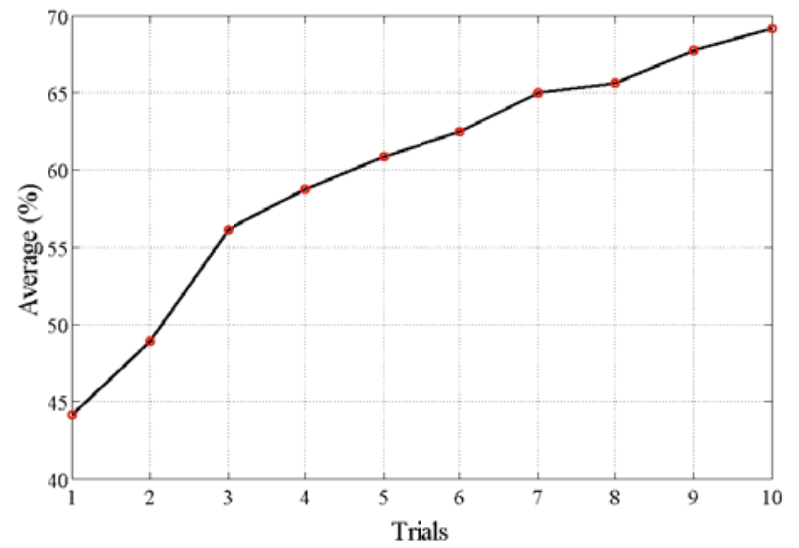

Fig. 8 Robot's performance in 10 experiments.

Table 4 Additional information of scenario4.

\begin{tabular}{llllccc}
\hline Test & \#hits & Cases & $\phi$ & $\theta$ & $\varphi$ & $\omega$ \\
\hline 1 & 20 & 321 & 11,518 & 988 & 1,064 & 1,092 \\
2 & 27 & 317 & 10,463 & 993 & 1,188 & 1,120 \\
3 & 29 & 314 & 11,512 & 1,034 & 983 & 1,114 \\
4 & 19 & 319 & 11,472 & 1,221 & 1,028 & 1,102 \\
5 & 19 & 318 & 11,632 & 1,198 & 1,311 & 1,023 \\
6 & 26 & 317 & 10,959 & 977 & 1,008 & 1,003 \\
7 & 23 & 320 & 11,476 & 1,103 & 1,182 & 992 \\
8 & 27 & 316 & 11,325 & 1,202 & 945 & 1,114 \\
9 & 28 & 312 & 10,953 & 1,153 & 1,122 & 973 \\
10 & 27 & 316 & 11,732 & 9,033 & 959 & 1,173 \\
AVE & 25 & 317 & 113,042 & 18,902 & 10,790 & 10,706 \\
\hline
\end{tabular}

of the robot's decision throughout the experiments. The complete analyzing results are summarized in Table 4. And then, Fig. 8 shows how the robot's performance increases in almost 36\% through the tests when the case-base has not been restarted. In particular, the robot's performance does not increase after seven experiments. This fact concludes that the case-base has reached a successful experience to solve any particular situation to avoid collisions in any particular indoor environment.

\section{Conclusions}

Experimental results indicate that the methodology proposed for mobile robot on two sonar rings to perform the navigation in an unknown, complex and changing indoor environment works in a proper way and can effectively solve the local trap problems in traditional mobile robot navigation strategy. Using 
CBR algorithm, the navigation status of a mobile robot is transferred when the information of environment changes, and a corresponding strategy is chose to realize the navigation task. In future, combination of a laser, vision sensors and other equipment will be used to reach a more complex mobile robot to autonomous navigation. The experiments report an average of 321 cases in the four testbeds. With the obtained results, it can be evaluated that the robot's performance is better when its experience is greater (i.e., when the casesbase contains the largest quantity of possible cases). For future work, the mapping task will be taken into account in order to endow mobile robot with a more suitable algorithm capable to avoid the robot to pass back through the same place.

\section{References}

[1] Wang, W., and Liu, J. N. K. 2008. "Fuzzy Logic-based Real-Time Robot Navigation in Unknown Environment with Dead Ends.”Journal Robotics and Systems, 56 (7):625-643.

[2] chai, T., Suksakulchai, S., Wilkes, D.M., and Sarkar, N. 2000. "Sonar Behavior-Based Fuzzy Control for a Mobile Robot.”In Proceedings of the IEEE International Conference on Systems Man and Cybernetics.

[3] Qian, K., and Song, A. 2012. "Autonomous Navigation for Mobile Robot Based on a Sonar Ring and Its Implementation, Instrumentation and Control
Technology."In Proceedings of the 8th IEEE International Symposium, 47-50.

[4] Jia, S. M., Shen, H. M.,Li, X. Z.,Cui, W., andWang, K. 2012. "Autonomous Robot Exploration Based on Hybrid Environment Model.”In Proceedings of the International Conference on Information and Automation, 19-24.

[5] Nichols, E., McDaid, L.J., and Siddique, N. 2013. "Biologically Inspired SNN for Robot Control, Cybernetics.”IEEE Transactions on 43 (1): 115-128.

[6] Zaman, S., Slany, W., and Steinbauer, G. 2011. "ROS-based Mapping, Localization and Autonomous Navigation Using a Pioneer 3DX robot and Their Relevant Issues, Electronics, Communications and Photonics Conference (SIECPC)."In Proceedings of theSaudi International, 1-5.

[7] Poncela, A., Urdiales, C., and Sandoval, F. 2007. “A CBR Approach to Behaviour-Based Navigation for an Autonomous Mobile Robot."In Proceedings of the IEEE International Conference on Robotics and Automation, 10-14.

[8] Aamodt, and Plaza, E. 1994. “Case-Based Reasoning: Foundational Issues, Methodological Variations, and System Approaches." AI Communications 7 (1): 39-59.

[9] Urdiales, C., Pérez, E.J., Vázquez-Salceda, J., Sánchez-Marré, M., and Sandoval, F. 2006. “A Purely Reactive Navigation Scheme for Dynamic Environments Using Case-Based Reasoning.” Autonomous Robots, 21: 65-78.

[10] Sánchez-Marré, M., Cortés, U., Béjar, J., Roda, I.R., and Poch, M. 1999. Reflective Reasoning in a Case-Based Reasoning Agent. vol. 1624. New York, NY: Springer-Verlag, 143-158. 